\title{
Scientific Exchange and Communication: Some Personal Views and Experiences
}

Text by David A. TIRRELL

I visited Japan for the first time thirty years ago, in the winter and spring of 1978. I was twenty-five years old, finishing my graduate work at the University of Massachusetts, and I was given the opportunity to study for four months in the laboratory of Professor Takeo Saegusa at Kyoto University. My Ph.D. supervisor, Professor Otto Vogl, had close ties to Professor Saegusa, and arranged the visit. Both personally and scientifically, it was a remarkable time for me.

My trip to Kyoto was my first real international experience, as I had never before left North America. I worked hard to learn the language, and although doing so was useful to me while I was in Japan, it has probably been even more useful in providing some rudimentary understanding of Japanese culture and in engendering empathy for those who must live and work in a second language. I developed an appreciation of the size and character of the Japanese polymer research community, and I established friendships that remain strong thirty years later. I've made a dozen trips to Japan over the intervening years and hosted more than twenty Japanese coworkers in my laboratories in Pittsburgh, Amherst and Pasadena. Each of those coworkers has made important contributions to our research in polymer chemistry. I currently have two Japanese colleagues in my laboratory, and I am looking forward to my next visit to Japan in December 2008.

Although I can't say that the Society of Polymer Science, Japan, has played a direct role in these interactions, organizations like SPSJ are essential to the overall health of our scientific communities. The remarkable strength of polymer research in Japan arises from many sources - an interest in education, a willingness to work hard, a commitment to providing the needed financial support-but the role of effective communication, as fostered by SPSJ and other scientific societies, should not be overlooked. Excellent journals, lively conferences, and full engagement with the international scientific community, are critically important to maintaining the vitality of science and technology in Japan and elsewhere.

With respect to the future, I would urge the leadership of SPSJ to think hard about how the Society can best contribute even further to improving scientific communication. The availability of electronic publications offers the prospect of revolutionary changes in the ways in which we communicate with one another, but I don't believe that we are yet taking full advantage of the opportunities that should be open to us. Most of us cannot read on our desktops many of the articles written by our colleagues, because our libraries do not subscribe to the journals where such articles are published. This situation is puzzling, given the enormous amount of information of other kinds that is freely available on the internet. I wouldn't argue that the solution to this problem is easy or obvious, but the problem is soluble and our professional societies (especially those that publish scientific journals) have a central role to play in finding a solution. I'm certain that imaginative people will see other opportunities to exploit electronic media in ways that go beyond traditional scientific publication.

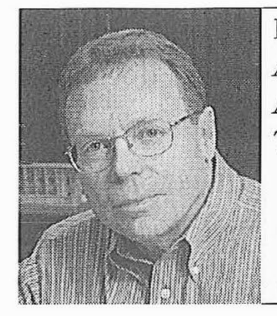

David A. Tirrell, Professor (tirrell@caltech.edu)

Advisory Editor and Former Editor, Journal of Polymer Science, Part A: Polymer Chemistry; Member, American Academy of Arts and Sciences. Division of Chemistry and Chemical Engineering, California Institute of Technology, Pasadena, CA 91125, U. S. A. 